\title{
Condition Monitoring of Single Point Cutting Tools Based on Machine Learning Approach
}

\author{
N. Gangadhar, Hemantha Kumar and S. Narendranath \\ National Institute of Technology Karnataka, Surathkal, Mangaluru-575025, India.
}

\author{
V. Sugumaran \\ SMBS, Vellore Institute of Technology University, Chennai Campus, Vandalur Kelambakkam Road, Chennai- \\ 600127, India.
}

(Received 15 August 2015; accepted 5 October 2016)

This paper presents the use of multilayer perceptron (MLP) for fault diagnosis through a histogram feature extracted from vibration signals of healthy and faulty conditions of single point cutting tools. The features were extracted from the vibration signals, which were acquired while machining with healthy and different worn-out tool conditions. Principle component analysis ( $P C A)$ used to select important extracted features. The artificial neural network $(A N N)$ algorithm was applied as a fault classifier in order to know the status of cutting tool conditions. The accuracy of classification with $M L P$ was found to be $82.5 \%$, which validates that the proposed approach is an effective method for fault diagnosis of single point cutting tools.

\section{NOMENCLATURE}

$\lambda \quad$ Eigen values

$\xi_{i} \quad$ Input vector

$\theta_{j} \quad$ Threshold of the $j^{\text {th }}$ neuron of the hidden layer

$u \quad$ Eigen vector

$A N N$ Artificial neural network

$B P \quad$ Back propagation

$D A Q \quad$ Data acquisition

$F P \quad$ False positive

$H S V$ Hue saturation value

$i \quad$ Input layer

j Hidden layer

$k \quad$ Output layer

$M L P$ Multilayer perceptron

$M S E$ Mean square error

$N I \quad$ National instruments

$P C A$ Principle component analysis

$T P \quad$ True positive

$W_{i j} \quad$ Weight vector connecting the $i^{\text {th }}$ neuron of the input layer to the $j^{\text {th }}$ neuron of the hidden layer

$W_{j k} \quad$ Weight vector connecting the $j^{t h}$ neuron of the hidden layer to the $k^{t h}$ neuron of the output layer

\section{INTRODUCTION}

Automated tool condition monitoring systems improves product quality and reduce defects and result in increased productivity. Automated tool monitoring systems prevents abrupt failure of the cutting tools and are extremely useful for modern automated machine tools. ${ }^{1}$ There are various machine learning techniques which have been applied for fault diagnosis in machinery systems. Ravikumar et al. used machine learning approaches for automated visual inspections of machine components. ${ }^{2}$ Sugumaran and Ramachandran studied the effects of a number of features on the classification of roller bearing faults, using both support vector machines and proximal support vector machines in their study. ${ }^{3}$ Indira et al. found a method for the calculation of optimum data and bin size of histogram features by employing fault diagnosis in a monoblock centrifugal pump. ${ }^{4}$ Taheri et al. used intelligent approach for a cooling radiator fault diagnosis, which was based on an infrared thermal image processing technique. ${ }^{5}$ Liu et al. presented a fog level detection method-based on an image hue saturation value (HSV) histogram - through an analysis of different $H S V$ information contained in different weather images. ${ }^{6}$ Sakthivel et al. reported the use of histogram features for decision treebased fault diagnosis in a monoblock centrifugal pump. ${ }^{7}$ Sugumaran and Ramachandran carried out fault diagnosis in roller bearing by using a fuzzy classifier and histogram features, with a focus on automatic rule learning. ${ }^{8}$

\subsection{Vibration Mode of a Turning Tool}

In a turning process, three different types of mechanical vibrations are present due to a lack of dynamic stiffness/rigidity of the machine tool system, which comprises the tool, tool holder, workpiece, and machine tool itself, as explained by Tobias. $^{9}$ These are free, forced, and self-excited chatter vibrations. Free vibrations are induced by shock and forced vibrations are due to an unbalanced effect in machine tool assemblies like gears, bearings, and spindles. Free and forced vibrations can be easily identified and eliminated. However, self-excited chatter vibrations are still not fully understood due to their complex nature. They are harmful for any machining process, including turning. Self-excited chatter vibrations are generally classified into either primary chatter or secondary chatter. ${ }^{10}$ Primary chatter is caused by friction between toolworkpiece, thermo-mechanical effects, or by mode coupling. Secondary chatter is caused by the regeneration of wavy surfaces on the workpiece. Regenerative vibration is the most destructive among the different vibrations. Bhuiyan et al. investigated tool wear, chip formation and the surface roughness of workpieces, each under different conditions while machining-, and used acoustic emission (AE) and vibration signature analysis in turning. ${ }^{11}$ They found that the AE and vibration com- 
ponents can effectively respond to different occurrences in tool wear and surface roughness. Sevilla et al. presented a reconfigurable system using the vibration signals generated from machining tests, which were performed under different tool conditions, and cut parameters for tool condition monitoring in the high-speed machining (HSM) process. ${ }^{12}$ Patra et al. developed a tool condition (flank wear) monitoring system using the vibration signals of the machining process. ${ }^{13}$ They showed that the fuzzy radial basis function based neural network can recognize the features extracted from the time domain by applying the wavelet packet approach, which underlies the vibration signals more effectively than other methods (e.g. back propagation neural network, radial basis function network, and normalized radial basis function network). Kilundu et al. integrated signal processing methodology and different machine learning methodologies (e.g. decision trees, Bayesian networks, k-Nearest Neighbour and neural network) to handle the computational complexities in monitoring the tool-wear by using cutting vibration signals. ${ }^{14}$ Jemielniak et al. studied the force, vibration, and acoustic emission signals while turning a specific material and extracting some features from the time, frequency, and time-frequency domains of signals detecting the tool wear. ${ }^{15}$ Scheffer and Heyns, reported the simultaneous use of vibration and strain measurement for wear monitoring in turning operation. ${ }^{16}$ Rao et al. analysed the workpiece roughness, vibration of the workpiece, and volume of the metal removal rate through a laser Doppler vibrometer and a high speed FFT analyser in a boring process. ${ }^{17}$ Rao and colleagues observed that the amplitude of vibration increased as the tool wear increased and that the feed rate was the significant parameter for affecting surface roughness. Scandiffio et al. investigated the influence of toolpath direction and tool-workpiece surface contact on the machining force, surface roughness, tool wear, and tool life in freeform milling by a ball end cutting tool when milling hard-quenched and tempered AISI D6 steel. ${ }^{18}$ They identified that the most influential factor for tool life was tool vibration.

\section{TOOL FAILURE MODES}

It is important to identify the different tool failure modes in order to select appropriate operating conditions for machining. The most widely studied tool failure modes are flank wear, breakage (fracture), crater wear, and plastic deformation. Only a few researchers reported tool failure because of notching (groove wear), cracking, and chipping. Notching and chipping changes the tool nose curvature. Figure 1, shows the tool failure modes, as depicted by Rao et al. ${ }^{19}$ Flank and crater wear are generally accepted as the normal tool failure modes, because the other failure modes can be avoided by selecting the proper machining parameters. The growth of flank and crater wear is directly related to the cutting time (or length of cut), unlike some of the other failure modes such as notching (groove wear), cracking, and chipping, which can occur unexpectedly, even with a new tool.

\subsection{Flank Wear}

Flank wear is mainly caused by the friction between the newly machined work piece surface and the tool flank face. Flank wear is marked on the cutting tool and is shown in Fig. 1a. It is responsible for a poor surface finish, a decrease

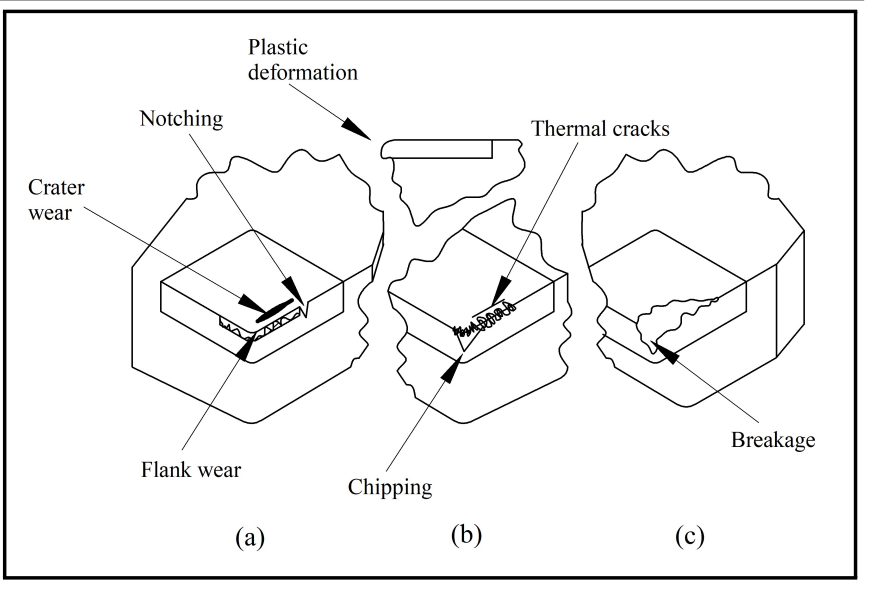

Figure 1. Different tool failure modes $(\mathrm{a}-\mathrm{c}) .{ }^{19}$

in the dimension accuracy of the tool, and an increase in cutting force, temperature, and vibration. The width of the flank wear-land is usually taken as a measure of the amount of wear, and the threshold value of the width is defined as a tool reshape criterion.

\subsection{Crater Wear}

Crater wear normally forms on rake face. It conforms to the shape of the chip's underside and reaches the maximum depth at a distance away from the cutting edge - where the highest temperature occurs. At high cutting speeds, crater wear is the main factor that determines the life of the cutting tool, due to a weakened tool edge which results in severe cratering and eventually fractures. Crater wear is improved by selecting suitable cutting parameters and using coated tools or ultra-hard material tools. Crater and flank wear are shown in Fig. 1a and are the most common wear types.

\subsection{Notch Wear}

Notch wear is a single groove formation that occurs simultaneously on the face and flank of the tool at the depth of the cut. Machining parts with severe (hard or oxidized) surfaces will cause notch wear. Figure 1a shows the depth of the cut due to notch wear.

\subsection{Chipping}

Figure 1b shows the chipping that occurred on the cutting edge. Chipping is the result of an overload of mechanical tensile stresses. These stresses can be due to a number of reasons, such as chip hammering, depth of cut or high feed rate, sand inclusions in the workpiece material, built-up edge, vibrations, or excessive wear on the insert.

\subsection{Thermal Cracks}

Thermal cracks appear on the rake face perpendicular to the cutting edge, as shown in Fig. 1b. Thermal cracking occurs when inserts go through rapid heating and cooling cycles. This failure mode is caused by interrupted cutting and by poor application of cutting fluids.

\subsection{Breakage}

Breakage is a mode of failure characterized by a breakaway of material on the tool edge. Breakage occurs when the feedrate is too high or when a tool is used with too low fracture 


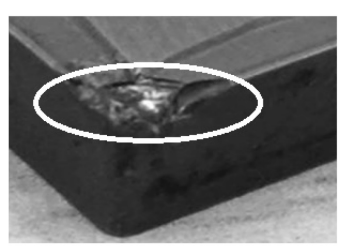

(i) Breakage

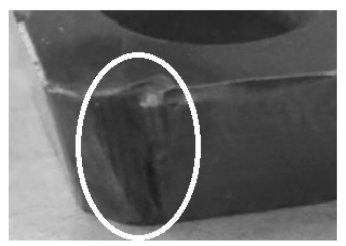

(iii) Notch wear

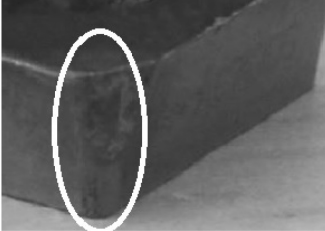

(ii) Thermal cracks

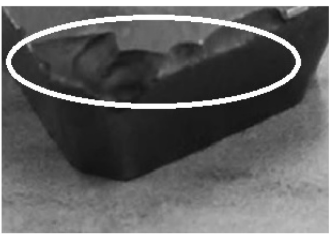

(iv) Rake face chipping
Figure 2. Different types of worn-out tool inserts.

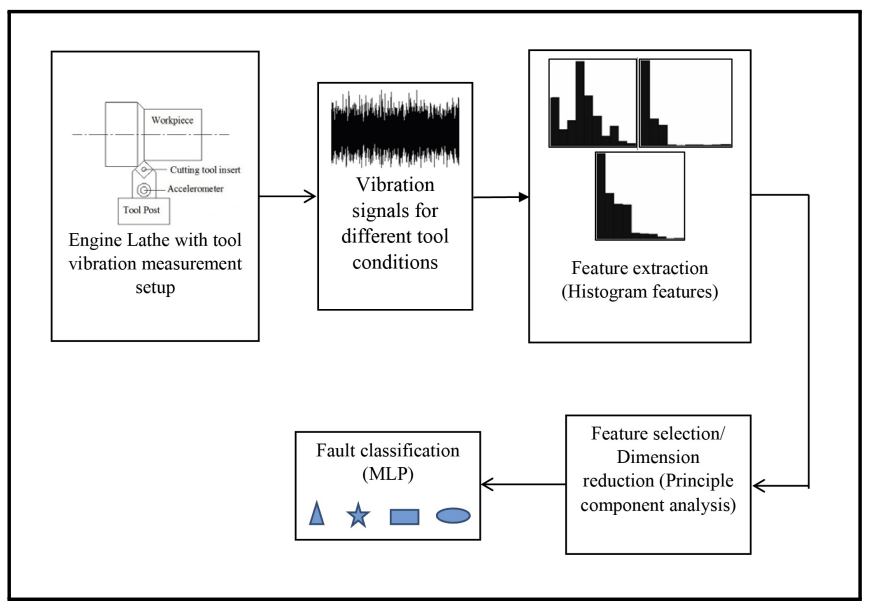

Figure 3. Flow chart of the fault diagnostic system.

strength. The usual pattern of wear of turning inserts is shown in Fig. 1c.

The different wornout conditions of the tool inserts considered in this present study are as follows: breakage, thermal cracks, notch wear, and rake face chipping, which are all shown in Fig. 2.

Vibration signals are widely used in the condition monitoring of rotating elements in machines. However, the classification of tool conditions for single point cutting tools using histogram features has not yet been attempted. In this present study, vibration signals were acquired with healthy and industrial wornout inserts. Histogram features were extracted from vibration signals to identify the status of the tool's condition. Fault detection is possible by comparing the signals of a machine running in both normal and faulty conditions. The methodology involved in the fault diagnosis of single point cutting tools, using a machine learning approach, is illustrated in Fig. 3. The vibration signals from the cutting tools, which were mounted on an engine lathe, was acquired using an accelerometer. Forty vibration signal samples were collected for each class of tool conditions. Histogram features were extracted from each of the collected samples using cboxMicrosoft Excel. The most important features out of those extracted were selected using principle component analysis $(P C A)$ as well as fault classification by use of multilayer perceptron $(M L P)$.

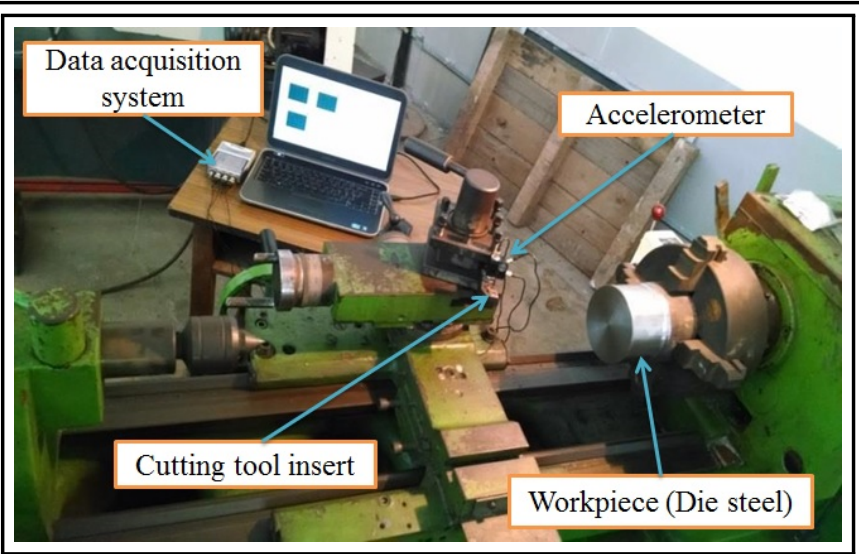

Figure 4. Schematic diagram of experimental setup.

\section{EXPERIMENTAL STUDIES}

The experiments were conducted on an engine lathe. Figure 4 shows a schematic diagram of the experimental setup. An accelerometer was used along with a data acquisition system for acquiring. A National Instruments $(N I)$ piezoelectric accelerometer and its accessories formed the core equipment for vibration measurement and recording. The output from the accelerometer was connected to the NI-9234 data acquisition $(D A Q)$ system and analysed by using LabVIEW software from $N I$. The vibration signals were acquired from single point cutting under healthy conditions (new) and by considering four different wornout inserts' conditions at a constant cutting speed of $236 \mathrm{~m} / \mathrm{min}$. The sampling frequency used in the study was $25.6 \mathrm{kHz}$ and each signal (sample) had a length of 25,600 data points. For each condition of the cutting tool, 40 samples were considered and recorded carefully.

One randomly selected vibration signal in time domain for each tool condition is shown in Fig. 5. From time domain signals, it was observed that the acceleration level increased with different fault conditions of cutting tools. A time domain technique for vibration signal analysis gave an overall vibration level, but it did not provide any diagnostic information.

\section{HISTOGRAM FEATURES}

A histogram is a graphical representation of the distribution of numerical data. To construct a histogram, the first step is to select data bin values, that is, divide the entire range of values into a series of small intervals and then count how many values fall into each interval. The histogram features were extracted from the time domain vibration signals. From the magnitude of the signal, it was found that the range varied from class to class. Corresponding histogram plots for different tool conditions are shown in Fig. 6.

The bin range should be from the lowest value of minimum amplitude (-20) to the highest value of maximum amplitude (+20) of all five classes. The number of bins for the fault diagnosis of single point cutting tools was obtained by carrying out a series of experiments using $M L P$ with a different number of bins. At first, range of bins was divided into two equal parts. That is to say, number of bins used was two. The two histogram features, namely $F_{1}$ and $F_{2}$, were extracted and the corresponding classification accuracy was also obtained by using $M L P$. A set of similar experiments were carried out with a different number of bins — from two, three, four ... 97 — and 


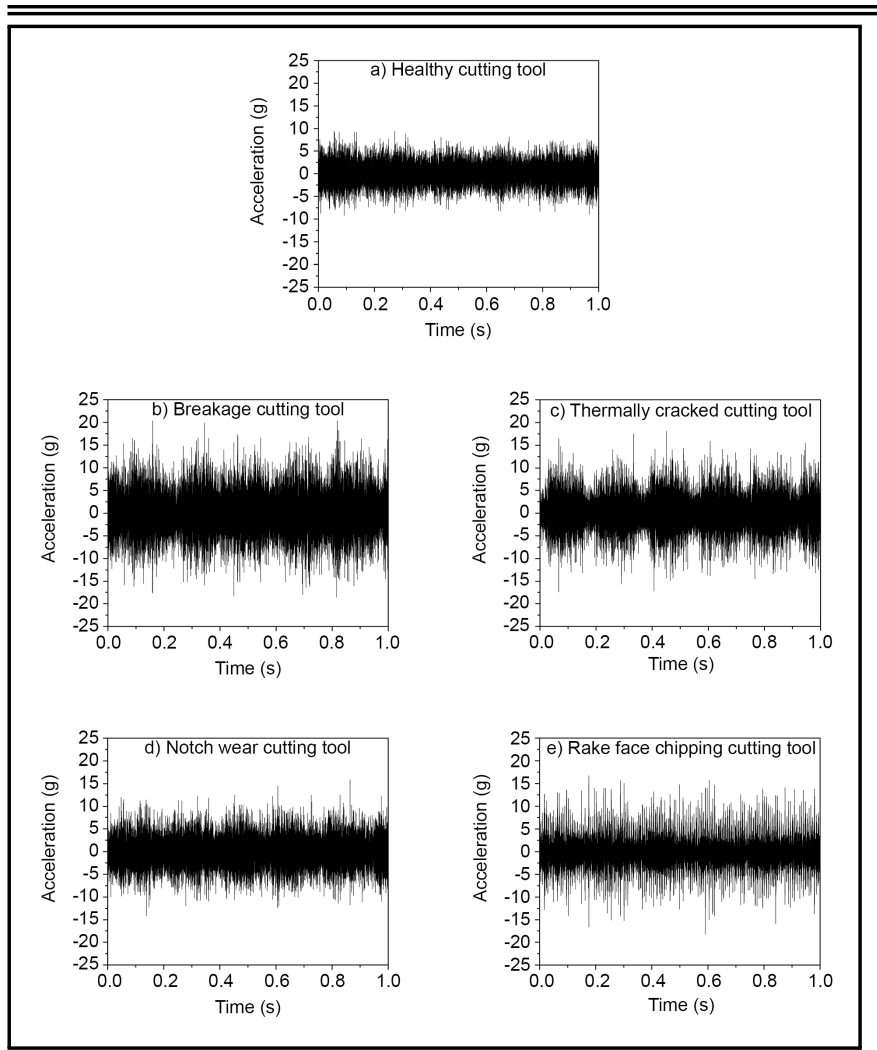

Figure 5. Acceleration observed with healthy and faulty cutting tool inserts in time domain.

the corresponding results are shown in Fig. 7. Upon careful observation of the results, the best classification accuracy of $94 \%$ is obtained when the number of bins is 15 , with a bin width of seven. Hence, these $F_{1}$ to $F_{15}$ histogram features were chosen as the bin parameters.

Table 1 shows the histogram features from $F_{1}$ to $F_{15}$. Out of 40 samples, only two samples pertaining to each class of tool condition are shown in Table 1. All extracted histogram features, $F_{1}$ to $F_{15}$ extracted from the vibration signals may not contain the information required for classification. The relevant features were selected using $P C A$.

\section{DIMENSIONALITY REDUCTION USING PRINCIPLE COMPONENT ANALYSIS}

Principal component analysis $(P C A)$ is one of the most widely used multidimensional features reduction tools. $P C A$ is the preferred choice because it is a simple and nonparametric method of extracting relevant information from complex data sets. The goal of $P C A$ is to reduce the dimensionality of the data while retaining as much as possible of the variation in the original data sets. Elangovan et al. discuss the use of $P C A$ with various classifiers - mainly to reduce the data dimensionality and report improvement in classifier efficiency. ${ }^{20} \mathrm{~A}$ similar approach for dimensionality reduction is attempted in this work. The basic workings of a $P C A$ are presented below:

Let $F_{1}, F_{2}, \ldots F_{n}$ be $N \times 1$ vectors.

Step 1: Mean value is calculated using the equation:

$$
\bar{F}=\frac{1}{N} \sum_{i=1}^{N} F_{i} .
$$

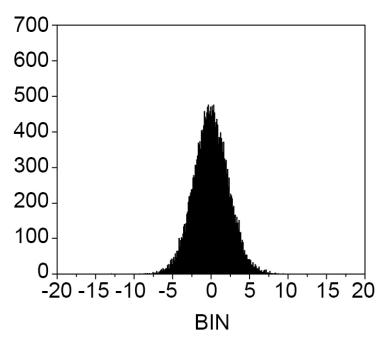

Healthy
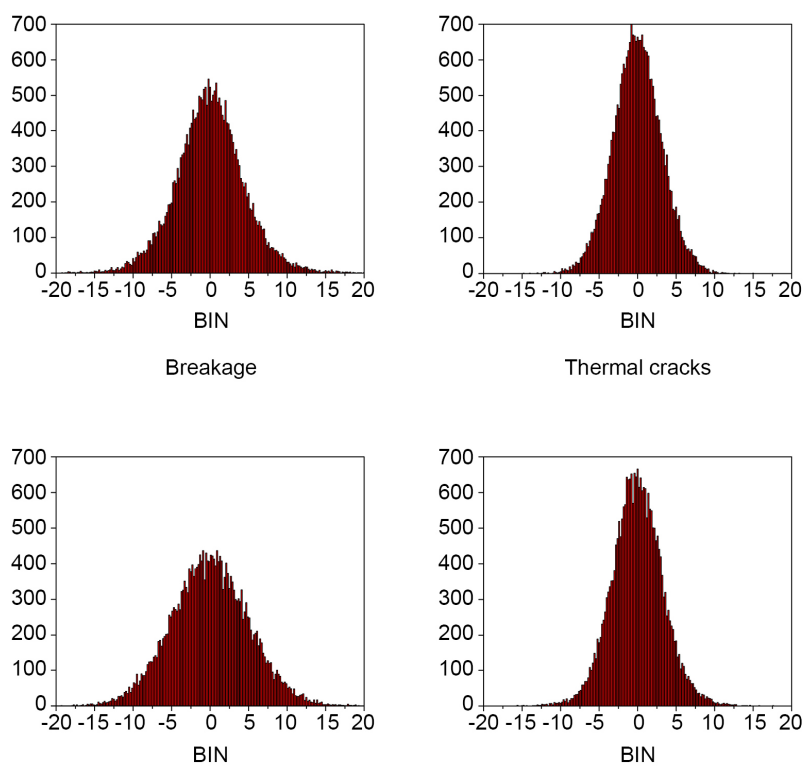

Notch wear

Rake face chipping

Figure 6. Histogram plots of signals.

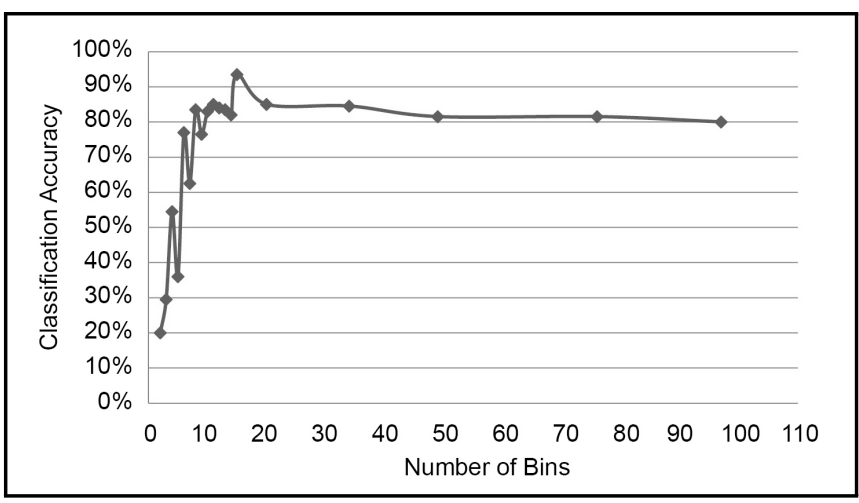

Figure 7. Number of bins vs. classification accuracy.

Step 2: Each feature is used to subtract the mean value:

$$
\phi_{i}=F_{i}-\bar{F} \text {. }
$$

Step 3: Matrix $A=\left[\phi_{1}, \phi_{2}, \ldots \phi_{N}\right]$ is generated by $N \times N$ matrix and covariance matrix $\mathrm{C}$ with the same dimension size, which is computed as follows:

$$
C=\frac{1}{M} \sum_{i=1}^{N} \phi_{i} \phi_{i}^{T}=A A^{T} .
$$

The covariance matrix characterizes the distribution of the data. Step 4: Eigenvalue is computed as:

$$
C=\lambda_{1}>\lambda_{2}>\cdots \lambda_{N}
$$


N. Gangadhar, et al.: CONDITION MONITORING OF SINGLE POINT CUTTING TOOL BASED ON MACHINE LEARNING APPROACH

Table 1. Histogram features extracted from the vibration signals for different tool conditions.

\begin{tabular}{|c|c|c|c|c|c|c|c|c|c|c|c|c|c|c|c|c|}
\hline \multirow{2}{*}{ Class } & \multirow{2}{*}{ Sample No. } & \multicolumn{15}{|c|}{ Histogram features } \\
\hline & & $F_{1}$ & $F_{2}$ & $F_{3}$ & $F_{4}$ & $F_{5}$ & $F_{6}$ & $F_{7}$ & $F_{8}$ & $F_{9}$ & $F_{10}$ & $F_{11}$ & $F_{12}$ & $F_{13}$ & $F_{14}$ & $F_{15}$ \\
\hline \multirow{2}{*}{ Healthy } & 1 & 0 & 0 & 0 & 0 & 0 & 0 & 599 & 12965 & 12036 & 0 & 0 & 0 & 0 & 0 & 0 \\
\hline & 2 & 0 & 0 & 0 & 0 & 0 & 0 & 809 & 12978 & 11813 & 0 & 0 & 0 & 0 & 0 & 0 \\
\hline \multirow{2}{*}{ Breakage } & 1 & 0 & 0 & 0 & 0 & 1 & 37 & 1507 & 11260 & 11220 & 1518 & 57 & 0 & 0 & 0 & 0 \\
\hline & 2 & 0 & 0 & 0 & 0 & 2 & 34 & 1190 & 11548 & 11596 & 1206 & 24 & 0 & 0 & 0 & 0 \\
\hline \multirow{2}{*}{ Thermal cracks } & 1 & 0 & 0 & 0 & 0 & 0 & 0 & 111 & 12651 & 12705 & 133 & 0 & 0 & 0 & 0 & 0 \\
\hline & 2 & 0 & 0 & 0 & 0 & 0 & 0 & 141 & 12843 & 12467 & 149 & 0 & 0 & 0 & 0 & 0 \\
\hline \multirow{2}{*}{ Notch wear } & 1 & 0 & 0 & 0 & 0 & 0 & 2 & 596 & 12279 & 12122 & 597 & 4 & 0 & 0 & 0 & 0 \\
\hline & 2 & 0 & 0 & 0 & 0 & 0 & 2 & 570 & 12212 & 12226 & 589 & 1 & 0 & 0 & 0 & 0 \\
\hline \multirow{2}{*}{ Rake face chipping } & 1 & 0 & 0 & 2 & 2 & 1 & 20 & 830 & 11944 & 11842 & 935 & 21 & 1 & 1 & 0 & 1 \\
\hline & 2 & 0 & 0 & 1 & 3 & 3 & 27 & 897 & 11865 & 11813 & 951 & 34 & 5 & 0 & 1 & 0 \\
\hline
\end{tabular}

Step 5: Eigenvector is computed as:

$$
C=\mu_{1}, \mu_{2}, \cdots \mu_{N}
$$

Since $\mathrm{C}$ is symmetric $\mu_{1}, \mu_{2}, \cdots \mu_{N}$ form a basis, and $\left(\left(F_{1}-\right.\right.$ $\bar{F})$ can be written as a linear combination of the Eigenvectors):

$$
F_{i}-\bar{F}=b_{1} u_{1}+b_{2} u_{2}+\cdots+b_{N} u_{N}=\sum_{i=1}^{N} l .
$$

Step 6: For dimensionality reduction, it keeps only the terms corresponding to the $\mathrm{K}$ largest Eigen values:

$$
F_{i}-\bar{F}=\sum_{i=1}^{K} b_{i} u_{i}, \text { where } K<<N .
$$

The representation of $E$. $E$ into the basis $u_{1}, u_{2}, \cdots u_{K}$ is thus,

$$
\left[\begin{array}{c}
b_{1} \\
b_{2} \\
\cdots \\
b_{K}
\end{array}\right] \text {. }
$$

\section{ARTIFICIAL NEURAL NETWORK}

An artificial neural network $(A N N)$ is modelled on biological neurons and nervous systems. ANN's have the ability to learn and have the processing elements known as neurons, which perform their operations in parallel. ANN's are characterized by their topology, weight vector, and activation functions. They have three layers: an input layer (which receives signals from the external world), a hidden layer (which does the processing of the signals), and an output layer (which gives the result back to the external world).

\subsection{Multilayer Perceptron (MLP)}

The $M L P$ is a unidirectional network, which has an input layer, hidden layer, and output layer, as shown in Fig. 8. The number of neurons in the input and output layers is equal to the number of input and output variables. Generally, one hidden layer is sufficient to map the problem in a correct way. The more complex the problem is: the more neurons are desirable.

As shown in Fig. 8, all input signals are received first by the input layer and then transmitted to other neurons in the hidden layer, where the processing task occurs. The information is then received by the output layer.

Each neuron in the hidden and output layer consists of an activation function, which is generally a nonlinear function like the logistic function, given by:

$$
f(x)=\frac{1}{1+e^{-x}}
$$

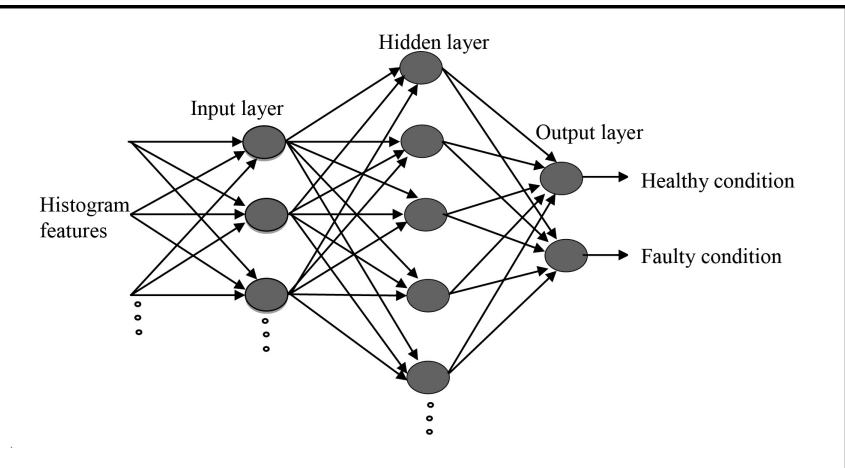

Figure 8. MLP network structure.

where $f(x)$ is differentiable and

$$
x=\sum_{i=1} W_{i j} \xi_{i}+\theta_{j}
$$

where $W_{i} j$ is the weight vector connecting the $i^{\text {th }}$ neuron of the input layer to the $j^{\text {th }}$ neuron of the hidden layer, and $\xi_{i}$ is the input vector and $\theta_{j}$ is the threshold of the $j^{\text {th }}$ neuron of the hidden layer. Similarly, $W_{j k}$ is the weight vector connecting $j^{\text {th }}$ neuron of the hidden layer with the $k^{\text {th }}$ neuron of the output layer: i-represents the input layer, j-represents the hidden layer, and k-represents the output layer. The weights that are important in predicting the process are unknown. The weights of the network to be trained are initialized to small random values. The choice of value selected obviously affects the rate of convergence. The weights are updated through an iterative learning process known as the Error Back Propagation $(B P)$ algorithm. The Error Back Propagation process consists of two passes through the different layers of the network — a forward pass in which input patterns are presented into the input layer of the network and its effect propagates through the network layer by layer. Finally, a set of outputs is produced as the actual response of the network. During the forward pass, the synaptic weights in the networks are all fixed. The error value is then calculated, which is the mean square error (MSE), given by

$$
E_{t o t}=\frac{1}{n} \sum_{n=1}^{n} E_{n}
$$

where $E_{n}=\frac{1}{2} \sum_{k=1}^{m}\left(\xi_{k}^{n}-O_{k}^{n}\right)^{2}$ and $m$ is the number of neurons in the output layer;

$\xi_{k}^{n}$ is the $k^{i h}$ component of the desired or target output vector and

$O_{k}^{n}$ is the $k^{i h}$ component of the output vector.

The weights in the links connecting the output and the hidden 
layer $W_{j k}$ are modified as follows:

$\Delta W_{j k}=\eta\left(-\partial E / \partial W_{j k}\right)=\eta \delta_{j} y_{j}$, where $\eta$ is the learning rate. Considering the momentum term $(\alpha) \Delta W_{j k}=\alpha \eta \delta_{j} y_{j}$ and $W_{j k}^{\text {old }}+\Delta W_{j k}$. Similarly, the weights in the links connecting the hidden and input layer $W_{j k}$ are modified as follows:

$$
\Delta W_{j k}=\alpha \eta \delta_{j} \xi_{j},
$$

where,

$$
\begin{gathered}
\delta_{j}=y_{j}\left(1-y_{j}\right) \sum_{k=1}^{m} \delta_{k} W_{j k}, \\
W_{i j}^{\text {new }}=W_{i j}^{\text {old }}+\Delta W_{i j}, \\
\delta_{k}=\left(\xi_{k}-O_{k}\right) O_{k}\left(1-O_{k}\right),
\end{gathered}
$$

for output neurons and for hidden neurons,

$$
\delta_{j}=y_{j}\left(1-y_{j}\right) \sum_{k=1}^{m} \delta_{k} W_{j k}
$$

The training process is carried out until the total error reaches an acceptable level (threshold). If $E_{t o t}<E_{\text {min }}$, the training process is stopped and the final weights are stored, which is used in the testing phase for determining the performance of the developed network. The sigmoid transfer function was used in the hidden and output layers.

\section{RESULTS AND DISCUSSIONS}

The histogram features discussed in section three are considered as features to serve as the input for the algorithm. The corresponding condition, or status, of the categorized data will be the essential output of the $M L P$ algorithm.

\subsection{Feature Extraction and Selection}

From the obtained vibration signals, 15 histogram features (f1, f2 ... f14 and f15) were extracted. The process of selecting relevant features is known as feature selection and it was carried out by using $P C A$.

These selected features serve the purpose of classification using the $M L P$ classifier algorithm. Classification accuracy of the $M L P$ classifier algorithm is presented in the form of a confusion matrix, as shown in Table 2. The meaning of confusion matrix is explained as follows:

In the first row, the first element shows the number of data points belonging to the 'healthy' class and classified by the classifier as 'healthy'. The total number of data points in the first row is $40 ; 31$ of them are correctly classified. In the first row, the other elements are zero except for in the fifth column, which means that nine of the healthy conditions are misclassified as rake face chipping.

The second row represents the total number of data points corresponding to the breakage condition; the first column represents misclassification of those data points as the healthy condition, which in this case is one. The second row, second column entry represents how many of the breakage samples are correctly classified as breakage by the classifier. Out of 40 samples, 37 are correctly classified. In this case, two samples are misclassified as the thermal crack condition. In the second row, other elements came out to zero. This means none of the breakage conditions are misclassified as notch wear, rake face chipping, and so on.
Misclassifications are among the healthy as well as faulty conditions, and they are about $17.5 \%$. However, the misclassification of healthy conditions as rake face chipping is about $4.5 \%$. The misclassification percentages of breakage as of healthy and thermal crack conditions are $0.5 \%$ and $1 \%$, respectively. The misclassification percentages of notch wear as breakage and rake face chipping conditions are $1.5 \%$ and $2 \%$, respectively. The misclassification percentages of rake face chipping as healthy, thermal cracks, and notch wear conditions are $3.5 \%, 0.5 \%$, and $4 \%$, respectively. The $M L P$ classifier performs absolutely well in classifying thermal crack samples. The performances differ slightly when classifying the healthy and faulty conditions. The misclassification in the $M L P$ classifier is $17.5 \%$. Out of 200 samples, 35 samples were incorrectly classified by the $M L P$, with a classification accuracy of $82.5 \%$ for vibration signals.

Table 3 shows the detailed class-wise accuracy of the multilayer perceptron. In Table 3, ' $T P$ rate' and ' $F P$ rate' are very important. The ' $T P$ rate' stands for true positive; its value should be close to ' 1 ' for better classification accuracy. The ' $F P$ rate' stands for false positive, and its value should be close to ' 0 ' for better classification accuracy. From the study, one can recognize the closeness of ' $T P$ rate' to ' 1 ' and ' $F P$ rate' to ' 0 '. Both values confirm that the model built is acceptable. From the obtained results, the classification accuracy can be appreciated.

\section{CONCLUSION}

This paper discusses the fault diagnosis of single point cutting tools using the machine learning approach based on vibration signals. This methodology involved collecting 40 acceleration vibration signal samples for five different classes of industrial wornout conditions. Histogram features were extracted from acquired vibration signals pertaining to all classes of fault categories. PCA was used for important feature selection. The $A N N$ algorithm was used for fault classification. Classification accuracy was found to be $82.5 \%$. Thus, $M L P$ classifier can be practically utilized to monitor the condition of tungsten carbide inserts while machining die steel.

\section{AKNOWLEDGEMENTS}

The authors acknowledge the Centre for System Design (CSD), a centre of excellence at NITK-Surathkal, for providing experimental facility.

\section{REFERENCES}

1 Wang, S. S. Artificial intelligence and expert systems for diagnostics, Proc. Institute of International Conference Machining and Diagnostics and Exhibition, Las Vegas, Nevada, (1989).

2 Ravikumar, S., Ramachandran, K. I., and Sugumaran, V. Machine learning approach for automated visual inspection of machine components, Expert Systems with Applications, 38 (4), 3260-3266, (2011).

3 Sugumaran, V., and Ramachandran, K. I. Effect of number of features on classification of roller bearing faults using SVM and PSVM, Expert Systems with Applications, 38 (4), 4088-4096, (2011). 
Table 2. Classification accuracy of the multilayer perceptron classifier.

\begin{tabular}{|c|c|c|c|c|c|}
\hline Healthy & Breakage & Thermal cracks & Notch wear & Rake face chipping & \\
\hline $\mathbf{3 1}$ & 0 & 0 & 0 & 9 & Healthy \\
\hline 1 & $\mathbf{3 7}$ & 2 & 0 & 0 & Breakage \\
\hline 0 & 0 & $\mathbf{4 0}$ & 0 & 0 & Thermal cracks \\
\hline 0 & 3 & 0 & $\mathbf{3 3}$ & 4 & Notch wear \\
\hline 7 & 0 & 1 & 8 & $\mathbf{2 4}$ & Rake face chipping \\
\hline
\end{tabular}

Table 3. Detailed accuracies by class.

\begin{tabular}{|c|c|c|c|c|c|c|}
\hline TP Rate & FP Rate & Precision & Recall & F-Measure & ROC Area & Class \\
\hline 0.775 & 0.05 & 0.795 & 0.775 & 0.785 & 0.946 & Healthy \\
\hline 0.925 & 0.019 & 0.925 & 0.925 & 0.925 & 0.951 & Breakage \\
\hline 1 & 0.019 & 0.93 & 1 & 0.964 & 0.992 & Thermal cracks \\
\hline 0.825 & 0.05 & 0.805 & 0.825 & 0.815 & 0.923 & Notch wear \\
\hline 0.6 & 0.081 & 0.649 & 0.6 & 0.623 & 0.861 & Rake face chipping \\
\hline
\end{tabular}

4 Indira, V., Vasanthakumari, R., Sakthivel, N. R., and Sugumaran, V. A method for calculation of optimum data size and bin size of histogram features in fault diagnosis of mono-block centrifugal pump, Expert Systems with Applications, textbf38 (6), 7708-7717, (2011).

5 Taheri-Garavand, A., Ahmadi, H., Omid, M., Mohtasebi, S. S., Mollazade, K., Smith, A. J. R., and Carlomagno, G. M. An intelligent approach for cooling radiator fault diagnosis based on infrared thermal image processing technique, Applied Thermal Engineering, 87, 434-443, (2015).

6 Liu, C., Lu, X., Ji, S., and Geng, W. A fog level detection method based on image HSV colour histogram, Proc. International Conference on Progress in Informatics and Computing (PIC) IEEE, Shanghai, China, (2014).

7 Sakthivel, N. R., Indira, V., Nair, B. B. and Sugumaran, V. Use of histogram features for decision tree-based fault diagnosis of monoblock centrifugal pump, International Journal of Granular Computing, Rough Sets and Intelligent Systems, 2 (1), 23-36, (2011).

8 Sugumaran, V., and Ramachandran, K. I. Fault diagnosis of roller bearing using fuzzy classifier and histogram features with focus on automatic rule learning, Expert Systems with Applications, 38 (5), 4901-4907, (2011).

9 Tobias, S. A. Machine tool vibration research, International Journal of Machine Tool Design and Research, 1 (1), 1-14, (1961).

10 Wiercigroch, M., and Budak, E. Sources of nonlinearities, chatter generation and suppression in metal cutting, Philosophical Transactions of the Royal Society of London a Mathematical, Physical and Engineering Sciences, 359 (1781), 663-693. (2001).

11 Bhuiyan, M. S. H., Choudhury, I. A. and Dahari, M. Monitoring the tool wear, surface roughness and chip formation occurrences using multiple sensors in turning, Journal of Manufacturing Systems, 33 (4), 476-487, (2014).

12 Sevilla-Camacho, P. Y., Robles-Ocampo, J. B., JaureguiCorrea, J. C. and Jimenez-Villalobos, D. FPGA-based reconfigurable system for tool condition monitoring in highspeed machining process, Measurement, 64, 81-88, (2015).
13 Patra, K., Pal, S. K. and Bhattacharyya, K. Fuzzy radial basis function (FRBF) network based tool condition monitoring system using vibration signals, Machining Science and Technology, 14 (2), 280-300, (2010).

14 Kilundu, B., Dehombreux, P. and Chiementin, X. Tool wear monitoring by machine learning techniques and singular spectrum analysis, Mechanical Systems and Signal Processing, 25 (1), 400-415, (2011).

15 Jemielniak, K., Urbaski, T., Kossakowska, J. and Bombiski, $\mathrm{S}$. Tool condition monitoring based on numerous signal features, The International Journal of Advanced Manufacturing Technology, 59 (1-4), 73-81, (2012).

16 Scheffer, C. and Heyns, P. S. Wear monitoring in turning operations using vibration and strain measurements, Mechanical Systems and Signal Processing, 15 (6), 11851202, (2001).

17 Rao, K. V., Murthy, B. S. N. and Rao, N. M. Cutting tool condition monitoring by analysing surface roughness, work piece vibration and volume of metal removed for AISI 1040 steel in boring, Measurement, 46 (10), 4075-4084, (2013).

18 Scandiffio, I., Diniz, A. E. and De Souza, A. F. Evaluating surface roughness, tool life, and machining force when milling free-form shapes on hardened AISI D6 steel, The International Journal of Advanced Manufacturing Technology, textbf82 (9-12), 2075-2086, (2015).

19 Rao, S. B. Tool wear monitoring through the dynamics of stable turning, Journal of Manufacturing Science and Engineering, 108 (3), 183-190, (1986).

20 Elangovan, M., Devasenapati, S. B., Sakthivel, N. R., and Ramachandran, K. I. Evaluation of expert system for condition monitoring of a single point cutting tool using principle component analysis and decision tree algorithm, Expert Systems with Applications, 38 (4), 4450-4459, (2011). 